\title{
Elementos de cambio y de continuidad en la militancia de ERC (1993-2004)
}

\section{Jordi Argelaguet}

Universitat Autònoma de Barcelona

Departament de Ciència Política i de Dret Públic

jordi.argelaguet@uab.cat

\section{Resumen}

En este artículo, se analiza la base humana del partido político catalán que se presenta como independentista: Esquerra Republicana de Catalunya. Para ello, se estudian los datos sociológicos de los asistentes a cuatro congresos nacionales del partido celebrados a lo largo del período 1993-2004: los de 1993, 1998, 2001 y 2004. La finalidad es plantear la profundidad de los vínculos - y el sentido de los mismos - entre base humana, oferta político-ideológica del partido y actuación pública en sus opciones de alianzas parlamentarias. A partir de los datos obtenidos, se traza el perfil del militante de ERC y se observan las pautas de cambio y de continuidad que ha habido a lo largo de estas casi dos décadas.

Palabras clave: partidos, Cataluña, nacionalismo, ERC, militantes.

\section{Abstract. The membership of ERC: elements of change and continuity (1993-2004)}

This article analyses the sociological profile the membership of the Catalan political party that presents itself as pro-independence, Esquerra Republicana de Catalunya. In order to do so, it offers data on the sociological profile of those attending four national party conferences over the 1993-2004 period: the conferences of 1993, 1998, 2001 and 2004. The overall aim is to analyse the strength and direction of the relationship between the party membership, the political and ideological position of the party, and the latter's parliamentary strategies. Based on such data, the article profiles the ERC membership and observes the trends of change and continuity over the period studied.

Key words: political parties, Catalonia, nationalism, ERC, members.

\section{Sumario}

1. Introducción 3. Las características sociodemográficas

2. Principales fases en la evolución de Esquerra Republicana de Catalunya de la militancia de ERC y sus opiniones políticas (1993-2004)

4. Conclusiones generales

Bibliografía 


\section{Introducción}

El estudio sobre los partidos políticos está presente en la ciencia política desde sus inicios como disciplina científica hace ya más de un siglo. Desde entonces, el conocimiento sobre estos importantes actores de la vida colectiva en las sociedades contemporáneas se ha incrementado substancialmente y se han generado multitud de hipótesis que se han contrastado con la realidad, a partir también de una diversidad considerable de enfoques metodológicos. A pesar del esfuerzo colectivo realizado, el análisis de los partidos políticos continúa siendo pertinente, porque quedan espacios para (re)explicar, entre otras razones, por ser una forma de organización que posee una alta capacidad para adaptarse a su entorno para poder cumplir sus funciones $y$, por lo tanto, se trata de una realidad en cambio constante, ya sea en sus estructuras o en sus lógicas de funcionamiento y de relación con otros partidos. Por todo ello, la afirmación que «una nueva mirada a los partidos resulta más pertinente que nunca» ${ }^{1}$ está plenamente justificada.

La presente nota de investigación se inscribe, lógicamente, en este marco del estudio de los partidos, pero está específicamente acotada, tanto por el ámbito concreto al que se orienta - el análisis sociológico de la base humana del fenómeno partidista-, como por el hecho que se trata de un estudio de caso - un partido menor en el sistema político español, por cuanto a sus votos o su número de militantes, pero con una proyección en la arena política nada desdeñable.

Así pues, en las páginas siguientes, se presentarán los datos sociológicos sobre la militancia de Esquerra Republicana de Catalunya (en adelante, ERC) que se han ido recabando en varios cuestionarios que se han ido pasando en congresos del partido realizados entre 1993 y 2004.

A través de la explotación y el análisis de los datos obtenidos, podemos apreciar cómo es la base humana de este partido y hasta qué punto ha ido cambiando a lo largo de esta última década, que ha coincidido con la reubicación de ERC en el espacio central de la política catalana y, en cierto sentido, de la española (Argelaguet, 2004). La finalidad última de esta aproximación al estudio de la militancia de ERC es plantear la profundidad de los vínculos - y el sentido de los mismos - entre base humana del partido, oferta político-ideológica y actuación pública en sus opciones de alianzas parlamentarias.

En efecto, un determinado conjunto de militantes segrega una determinada oferta política que puede atraer a nuevos militantes hacia la organización. Como resultado, el partido puede mutar en su base humana, la cual, a su vez, se dispone a publicitar su propia oferta política, que puede ser, o no, coincidente con la anterior. El partido deviene un ente cambiante que, si bien conserva elementos de continuidad mínimos, más allá del nombre, tales como unos principios ideológicos, unos referentes históricos, un liderazgo e, incluso, una determinada «cultura política» propia de la organización, debe de ser apre- 
hendido como tal, dinámico y fluyente. De hecho, de la misma forma que se atribuye a Heráclito la expresión "panta rei», para encapsular la idea que es imposible bañarse dos veces en el mismo río, en el caso de los partidos, podría decirse que es imposible votar dos veces al mismo partido, ya que es diferente que lo que fue y de lo que será.

\section{Principales fases en la evolución de Esquerra Republicana de Catalunya}

La larga trayectoria de Esquerra Republicana de Catalunya desde su fundación, en marzo de 1931, hasta la actualidad, ha generado una tal gran multitud de literatura de estudios y análisis, que es improcedente, ahora y aquí, reproducir ${ }^{2}$. No obstante, puede ser útil resumirla a través de la identificación de grandes etapas en la vida del partido: la etapa republicana (1931-1939), la resistencia al franquismo (1939-1976) y la del restablecimiento de la democracia y la autonomía de Cataluña (desde 1977 hasta la actualidad).

Dentro de esta última etapa, la evolución electoral de ERC ha variado bastante. Ha pasado de ser un partido menor y casi marginal, a ser la tercera fuerza política en Cataluña (ver tabla 1).

Además, en esta última etapa democrática, se identifican tres grandes subfases, coincidentes con el mandato de otros tantos secretarios generales y sus subsiguientes planteamientos políticos: Heribert Barrera (1976-1987), Àngel Colom (1989-1996) y Josep-Lluís Carod-Rovira (desde 1996 hasta la actualidad). Cabe decir, no obstante, que ha habido un cuarto secretario general, Joan Hortalà, quien substituyó a Barrera y sirvió de transición, a su pesar, hasta la llegada al poder de Colom.

Los cambios en el liderazgo han tendido a coincidir con los cambios en la ideología formalmente adoptada por el partido. En efecto, la propuesta ideológica de ERC se sustenta en los «Principios básicos», aprobados por la Asamblea de Constitución del partido en junio del 1931. Estos principios fueron puestos al día por la "Declaración ideológica", aprobada en el X Congreso Extraordinario celebrado en Barcelona en el 16 de diciembre de 1979 y en el 20 de enero de 1980. Ambos textos fueron completados con la «Declaración ideológica», aprobada por el XIX Congreso Nacional celebrado en Barcelona en el 19 de diciembre de 1993. Este congreso significó la concreción del giro independentista del partido que se había impuesto en el XVI Congreso, celebrado en Lleida en 1989. En aquel congreso, la militancia de ERC eligió en segunda votación a Àngel Colom como secretario general frente a Joan Hortalà 3 .

Las consecuencias del traumático congreso de Lleida fueron no sólo el replanteamiento de ERC como partido abiertamente independentista, sino también la culminación de una maniobra de entrismo que había empezado un

2. Las referencias básicas son las siguientes: Alquézar y otros (2001), Ivern (1989), Sallés (1986). En menor medida, también pueden ser útiles Molas (ed.) (2000) y Lucas (2004).

3. En la primera votación, Hortalà obtuvo 879 votos; Colom, 772, y Carod-Rovira, 235. En la segunda votación, Colom obtuvo 920 votos, y Hortalà, 886. 
Tabla 1. Resultados electorales de ERC y su representación política

\begin{tabular}{llcccr}
\hline & & & \multicolumn{2}{c}{ Votos válidos \% } & \\
\cline { 3 - 5 } Año & Elecciones & Votos en Cataluña & De Cataluña & De España & Escaños \\
\hline 1977 & Españolas & 143.954 & 4,7 & 0,8 & 1 \\
1979 & Españolas & 123.452 & 4,2 & 0,7 & 1 \\
1979 & Locales & 103.547 & 3,9 & No disp. & 210 \\
1980 & Catalanas & 240.871 & 8,9 & - & 14 \\
1982 & Españolas & 138.118 & 4,0 & 0,7 & 1 \\
1983 & Locales & 84.984 & 2,9 & No disp. & 155 \\
1984 & Catalanas & 126.943 & 4,1 & - & 5 \\
1986 & Españolas & 85.063 & 2,7 & 0,4 & 0 \\
1987 & Locales & 74.700 & 2,5 & 0,4 & 185 \\
1988 & Catalanas & 111.647 & 4,1 & - & 6 \\
1989 & Españolas & 85.137 & 2,7 & 0,4 & 0 \\
1991 & Locales & 91.995 & 3,4 & 0,5 & 228 \\
1992 & Catalanas & 210.366 & 8,0 & - & 11 \\
1993 & Españolas & 187.573 & 5,1 & 0,8 & 1 \\
1995 & Locales & 202.242 & 6,3 & 0,9 & 526 \\
1995 & Catalanas & 305.867 & 9,5 & - & 13 \\
1996 & Españolas & 163.341 & 4,2 & 0,7 & 1 \\
1999 & Locales & 224.955 & 7,7 & 1,1 & 671 \\
1999 & Catalanas & 271.173 & 8,7 & - & 12 \\
2000 & Españolas & 191.218 & 5,6 & 0,8 & 1 \\
2003 & Locales & 414.549 & 12,8 & 1,8 & 1.278 \\
2003 & Catalanas & 544.324 & 16,4 & - & 23 \\
2004 & Españolas & 642.112 & 15,9 & 2,5 & 8 \\
2006 & Catalanas & 416.355 & 14,0 & - & 21 \\
2007 & Locales & 334.771 & 11,7 & 1,5 & 1.584 \\
2008 & Españolas & 291.532 & 7,8 & 1,1 & 3 \\
\hline 1.25 & & & &
\end{tabular}

1. Resultados para el Congreso de los Diputados.

Fuentes: para las eleccions legislativas españolas, Ministerio del Interior; para las legislativas catalanas o las locales, Generalitat de Catalunya; para las elecciones locales de 2007, Ministerio del Interior.

par de años antes, encabezada por Àngel Colom, Josep-Lluís Carod-Rovira y Joan Puigcercós, a raíz de un manifiesto publicado a inicios de 1987. Además, el secretario general saliente, Joan Hortalà, juntamente con algunos militantes y cargos electos ${ }^{4}$, impulsó una nueva formación política, Esquerra Catalana, que, al cabo de unos años, acabó disuelta y con la mayoría de sus miembros afiliados a CDC. En noviembre de 1989, pues, ERC se refundó sobre un legado histórico, pero con una propuesta política renovada que se concretó en los congresos nacionales de 1991 (con la elección de Heribert Barrera como pre-

4. Básicamente, dos diputados del Parlamento de Cataluña. Juntamente con Hortalà, significaban la mitad del grupo parlamentario de ERC en aquella legislatura. 
sidente «honorífico» del partido), de 1992 (con la aprobación de los nuevos estatutos del partido que establecían formalmente que el objetivo de ERC era la independencia de Cataluña y que su ámbito de actuación era todo el territorio de los llamados «Països Catalans») y de 1993 (con la aprobación de la ya mencionada «Declaración ideológica»).

El mandato de Àngel Colom se desarrolló en este marco hasta 1996, cuando varios sectores del partido, descontentos con la marcha del mismo (en términos internos y electorales) y con los planteamientos ideológicos que transmitía, se empezaron a movilizar hasta conseguir la salida del partido del secretario general y de sus seguidores. En octubre de 1996, Colom, algunos cargos electos, algunos cuadros y centenares de militantes abandonaron ERC para formar el Partit per la Independència. Este partido fracasó en las urnas de las siguientes elecciones (las locales de 1999) y se disolvió. Muchos de sus ya exmilitantes, con Colom en la cabeza, ingresaron en Convergència Democràtica de Catalunya (CDC).

Por su lado, en 1996, los nuevos dirigentes de ERC — todos ellos antiguos colaboradores de Colom desde 1987 hasta 1989- ocuparon las responsabilidades orgánicas en el partido y formalizaron la toma del poder en el XXI Congreso Nacional, celebrado en Vilafranca del Penedès en noviembre de aquel mismo año. Desde entonces, el «nuevo» liderazgo de ERC impuso un giro izquierdista, bajo el lema de la «izquierda nacional», que le condujo a reorganizar primero el partido; mejorar después los resultados electorales, y, como corolario de su actuación, construir una coalición de gobierno con el PSC-PSOE e ICV después de las elecciones al Parlamento de Cataluña de 2003. Con esta decisión, ERC, que también podía constituir mayoría parlamentaria con CiU, ponía fin a veintitrés años de gobierno del catalanismo moderado en la Generalitat de Catalunya.

En paralelo a los cambios en los planteamientos políticos públicos del partido, en su liderazgo, en sus resultados electorales o en su estructura interna, ERC ha registrado también cambios en sus bases humanas, es decir, en cuantos son los miembros de ERC y en como son.

Para hacer una aproximación a estos datos relativos a la base humana de ERC, en cuanto a la magnitud del número de sus miembros, puede ser útil referirse a los niveles de participación en los congresos nacionales, así como en las votaciones más significativas de los mismos. Todos estos datos se han reproducido en la tabla 2 .

Desde la transición, los dirigentes de ERC habían manifestado, públicamente y habitualmente, que el partido contaba con unos 6.000 o 7.000 militantes. A pesar de que estas cifras no podían ser contrastadas al margen del partido, el número de votaciones registradas en los diferentes congresos (así como las referencias en la prensa sobre los asistentes) permite sospechar que la cantidad de militantes de ERC debía de ser bastante inferior. Entre 1976 y 1989, en los congresos de ERC, se admite tanto la participación individual de cualquier militante como la delegación de voto. Por todo ello, es de esperar que la votación final no represente sólo a los asistentes, sino también al total de 
Tabla 2. Votos en la elección del secretario general de ERC en los congresos nacionales (1976-2004)

\begin{tabular}{|c|c|c|c|c|c|}
\hline Año & Núm. & Lugar & Fecha & $\begin{array}{l}\text { Votos en la elección } \\
\text { del secretario general }\end{array}$ & $\begin{array}{l}\text { Número asistentes } \\
\text { (aprox.) }\end{array}$ \\
\hline 1976 & 8 & Barcelona & 4 de julio & Por aclamación & Unos 500 \\
\hline 1977 & 8 (extra.) & Barcelona & 2 de abril & Por aclamación & 600 \\
\hline 1979 & 9 & Barcelona & $\begin{array}{r}17 \text { y } 18 \\
\text { de diciembre }\end{array}$ & No disponible & Unos 500 \\
\hline 1979 & 10 & Barcelona & $\begin{array}{l}17 \text { de junio- } \\
1 \text { de julio }\end{array}$ & 1.748 & Unos 500 \\
\hline 1979 & 10 (extra.) & Barcelona & $\begin{array}{r}16 \text { de diciembre- } \\
20 \text { de enero }\end{array}$ & No elección SG & Unos 800 \\
\hline 1980 & 11 & Barcelona & $\begin{array}{r}18 \text { y } 19 \\
\text { de octubre }\end{array}$ & No disponible ${ }^{1}$ & Unos 1.000 \\
\hline 1981 & 12 & Barcelona & $\begin{array}{r}5 \text { y } 6 \text { de } \\
\text { diciembre }\end{array}$ & 880 & No disponible \\
\hline 1983 & 13 & Barcelona & 18 y 19 de junio & 2.424 & Unos 2.000 \\
\hline 1985 & 14 & Barcelona & 19 y 20 de enero & 799 & 300 \\
\hline 1987 & 15 & Barcelona & 24 y 25 de enero & 1.040 & No disponible \\
\hline 1989 & 16 & Lleida & $\begin{array}{l}18 \text { y } 19 \text { de } \\
\text { noviembre }\end{array}$ & $1.806^{2}$ & Unos 750 \\
\hline 1991 & 17 & Barcelona & 15 de diciembre & 813 & No disponible ${ }^{3}$ \\
\hline 1992 & 18 & Vic & 27 y 28 de junio & No elección SG & Unos 400 \\
\hline 1993 & 19 & Barcelona & $\begin{array}{l}18 \text { y } 19 \text { de } \\
\text { diciembre }\end{array}$ & 841 & \\
\hline 1995 & 20 & Manlleu & 9 de julio & 1.163 & \\
\hline 1996 & 21 & Vilafranca & $\begin{array}{l}23 \text { y } 24 \text { de } \\
\text { noviembre }\end{array}$ & 987 & \\
\hline 1998 & 22 & Girona & 4 y 5 de julio & 537 & \\
\hline 2001 & 23 & Tarragona & 17 y 18 de marzo & 777 & \\
\hline 2004 & 24 & Lleida & 3 y 4 de julio & $1.362^{4}$ & \\
\hline 2008 & 25 & Barcelona & 14 de julio & $2.722^{5}$ & \\
\hline
\end{tabular}

1. En la votación sobre estatutos, se emitieron 1.264 votos.

2. En la primera votación para la secretaría general, se emitieron 1.886 votos.

3. A partir de este congreso, no se permite el voto delegado.

4. En la votación para los consejeros nacionales, se emitieron 1.379 votos.

5. En este congreso, los militantes de ERC habían votado, la semana anterior, en sesenta colegios electorales establecidos a tal efecto, para elegir, directamente y por separado, al presidente y al secretario general del partido. En estas elecciones, participaron 7.036 votantes (el 70,9\% del censo) y resultó elegido presidente Joan Puigcercós, con 2.616 votos. En el congreso, propiamente dicho, la elección con más participación (2722) fue la de los consejeros nacionales. Para asistir a este congreso, se habían inscrito 4.043 militantes. 
los votos delegados. La votación más numerosa registrada se dio en el XIII Congreso (en 1983), cuando se contaron 2.424 votos. La segunda más numerosa fue en 1989, en el ya citado congreso celebrado en Lleida y en el que todos los militantes sabían qué se dirimiría allí. Por esta razón, se tiene que pensar que la gran mayoría de la militancia o bien estuvo en directo o bien delegó su voto. Aún así, la votación más numerosa registró un total de 1.886 votos.

A partir de los estatutos aprobados en 1992 (en el XVIII Congreso, en Vic), se suprimió la delegación de voto. Desde aquel momento, en los congresos, cada participante tenía un solo voto y se consolidó la práctica asamblearia de los congresos de ERC. De esta forma, desde 1992 hasta la actualidad, las votaciones en los congresos sólo indican —-más o menos- el número de participantes en ellos, que se autootorgan la representación del conjunto de la militancia, si bien sólo acostumbran a ser una minoría que no excede, en el mejor de los casos, el 27\% del total de militantes de ERC (según los datos del congreso de 2008).

Las cifras de la militancia de ERC, para fechas más recientes, parecen ser más fiables y más ajustadas a la realidad, ya que el propio partido ha estado haciendo un esfuerzo para depurar su censo, debido a las necesidades de gestionar bien sus recursos económicos y, también, como exigencia de la transparencia democrática mínima en un partido que basa el funcionamiento de sus congresos en una asamblea de militantes al corriente de pago.

A partir del Congreso de Lleida (1989), los datos de militancia disponibles son los que se reproducen en la tabla $3^{5}$.

Como se aprecia, desde 1989 hasta 2006, ERC ha multiplicado por cuatro su militancia, al pasar de los casi 2.500 miembros en el Congreso de Lleida, a los más de 10.000 en abril de 2008. La evolución no es, sin embargo, siempre ascendente. Desde 1989 hasta 1996, el partido no deja de crecer, pero, a raíz de los «hechos de octubre» de 1996 (que se saldaron con la escisión del Partit per la Independència), el partido sufrió una pérdida considerable de miembros. Durante la segunda mitad de la década de 1990, muy lentamente, la agrupación política empezó a incrementar su militancia. A finales del 2003, coincidiendo con su mejor resultado en unas elecciones del Parlamento de Cataluña en la etapa monárquica, ERC superó la barrera de los 6.000 militantes que había tenido una década antes. Con la entrada en el gobierno de la Generalitat, la militancia de ERC se disparó y, en tres años, alcanzó la cifra de los 10.000 militantes. Desde entonces, el crecimiento neto de ERC casi se ha estancado. En efecto, si bien han entrado en el partido más de 1.500 personas, en abril de 2008 había sólo 129 militantes más que en octubre de 2006.

5. La Secretaría de Organización de ERC cuenta con un notable archivo de toda la militancia del partido en estos años, estructurado de tal forma que incluso permite un cierto tratamiento estadístico detallado. Para esta nota de investigación, no se ha tenido acceso directo a esta base de datos, pero sí a varios documentos internos (pero oficiales) del partido que dan cuenta de la evolución de la militancia de ERC de una manera rigurosa. 
Tabla 3. Número de militantes (1989-2006)

\begin{tabular}{|c|c|c|c|}
\hline Año & Número & Fecha & Fuente \\
\hline 1989 & 2.489 & Noviembre. & $\begin{array}{l}\text { Informe del secretario general (Àngel Colom) } \\
\text { en el Consejo Nacional del } 28 \text { de noviembre de } \\
1992 .\end{array}$ \\
\hline 1992 & 5.525 & Noviembre $^{1}$. & $\begin{array}{l}\text { Informe del secretario general (Àngel Colom) } \\
\text { en el Consejo Nacional del } 28 \text { de noviembre de } \\
1992 .\end{array}$ \\
\hline 1996 & 6.530 & 3 de mayo. & $\begin{array}{l}\text { Documento interno para la reunión de Santa } \\
\text { Pau. }\end{array}$ \\
\hline 1996 & 6.900 & 30 de junio. & Documento precongresual de 1996. \\
\hline 1997 & 6.094 & Marzo. & Presupuesto de ERC de marzo de 1997. \\
\hline 1998 & 4.582 & Julio. & $\begin{array}{l}\text { Ponencia de la organización del XXII Congreso } \\
\text { Nacional. }\end{array}$ \\
\hline 2001 & 4.420 & 1 de enero. & $\begin{array}{l}\text { Ponencia de la organización del XXIV } \\
\text { Congreso Nacional. }\end{array}$ \\
\hline 2001 & 4.820 & Diciembre. & Liquidación del presupuesto de 2002. \\
\hline 2002 & 4.934 & 30 de junio. & Esquerra Nacional, 35. \\
\hline 2002 & 5.478 & Diciembre. & Liquidación del presupuesto de 2002. \\
\hline 2003 & 7.067 & Diciembre. & Esquerra Nacional, 51 \\
\hline 2004 & 8.614 & Marzo $^{5}$ & $\begin{array}{l}\text { Ponencia de la organización del XXIV } \\
\text { Congreso Nacional. }\end{array}$ \\
\hline 2006 & 10.000 & 7 de octubre. & ERC. Comunicado de prensa. \\
\hline 2008 & 10.129 & Abril ${ }^{6}$ & Esquerra Nacional, 91. \\
\hline
\end{tabular}

1. El 73,74\% de los militantes (4.074) estaba al corriente de pago de las cuotas.

2. El 69,16\% de los militantes (3.169) estaba al corriente de pago.

3. Militantes al corriente de pago.

4. Militantes al corriente de pago.

5. El 94,14\% de los militantes (8.109) estaba al corriente de pago.

6. El censo para la elección del presidente y del secretario general en el marco del XXV Congreso de ERC era de 9.923 personas.

Para calibrar mejor la magnitud de estas cifras, puede ser útil recurrir a la ratio entre votantes y militantes de ERC. Para las elecciones catalanas de 2003, esta ratio era de 77,02 votantes por militante, y para las elecciones españolas de 2004, la ratio era de $74,5^{6}$. Expresando esta ratio en porcentaje de los militantes en relación con los votantes, para las elecciones legislativas españolas, ERC presentaba un porcentaje del 1,34\%, que contrasta enormemente con el 6,83\% del PP, el 4,96\% del PSOE o el 5,28\% de IU, para el mismo año (Verge,

6. En 2003, los votos de ERC fueron 544.324 y los militantes eran 7.067. En 2004, los votos fueron 642.112 y los militantes eran 8.614 . 
Tabla 4. Número de altas por año (1990-2008)

\begin{tabular}{rrrr}
\hline Año & Número altas & Año & Número altas \\
\hline 1990 & 568 & 2000 & 609 \\
1991 & 1.069 & 2001 & 592 \\
1992 & 1.500 & 2002 & 1.041 \\
1993 & 1.113 & 2003 & 1.985 \\
1994 & 586 & 2004 & 2.954 \\
1995 & 884 & 2005 & 902 \\
1996 & 815 & 2006 & 1.096 \\
1997 & 219 & 2007 & 1.177 \\
1998 & 225 & $2008^{1}$ & 573 \\
1999 & 594 & Total altas & $\mathbf{1 8 . 5 0 2}$ \\
\hline
\end{tabular}

Fuente:

Para el período 1990-2003, Esquerra Nacional, número 51, enero de 2004.

Para los años 1998-2008, elaboración propia a partir de los datos publicados periódicamente en Esquerra Nacional.

En Esquerra Nacional, desde el número 6 (julio-agosto de 1998) hasta el número 66 (octubre de 2005), se publican los nombres de todas las solicitudes de afiliación que llegan al partido, así como la sección local donde se adscriben.

1. Hasta abril de 2008.

2007: 211). La explicación de estas diferencias escapa al propósito de la presente nota de investigación. No obstante, se podrían plantear dos alternativas. Asumiendo la veracidad de las cifras de ERC, o bien el bajo porcentaje de militantes de ERC en relación con los votantes obedece a que se trata de un partido antisistema (por independentista, de izquierdas y republicano), por lo que el hecho de militar en él no deja de ser un poco arriesgado, o bien porque las cifras de militancia de los otros partidos están decididamente hinchadas.

Finalmente, para concluir este repaso a la evolución de la militancia de ERC en estos últimos años, en la tabla 4 se recoge el número de altas anuales que el partido ha registrado entre 1990 y 2007.

De la tabla 4, se puede apreciar cómo se ha desarrollado el proceso de captación de militancia hacia ERC durante estos últimos años y la relación temporal que se establece con los avatares político-electorales que el partido vive ${ }^{7}$. De la misma forma, cabe resaltar que, en diecisiete años y cuatro meses, ERC ha recibido 18.502 solicitudes de alta, de las cuales el 36,2\% (6.702) se han producido después de la decisión de haber constituido el gobierno tripartito con los socialistas y postcomunistas en la Generalitat de Catalunya.

Finalmente, dado que el partido contaba con 2.489 miembros en el año cero (1989) de esta serie, dado que se han registrado 17.860 altas, cuando el partido dice tener poco más de 10.000 militantes, todo indica que las bajas tam-

7. Se tiene que señalar que se trata de solicitudes de alta en el partido y que puede darse la circunstancia que una persona haya militado en él en dos o más ocasiones, pero con periodos de baja en medio. Cada vez que pide el reingreso en el partido, cuenta una nueva alta. 
bién han sido cuantiosas. Dejando de lado las bajas por defunción, el resto expresaría un cierto alejamiento de la formación política que no puede ser obviado. Sea como sea, las grandes cifras indicarían un determinado efecto de revolving door, es decir, se trata de una organización de la cual constantemente están entrando y saliendo afiliados.

Aunque sería interesante abordar un estudio detallado de quiénes son y cómo son las personas que entran y salen de un partido, los datos por ahora disponibles sólo nos permiten ver algunos fotogramas de este filme. En efecto, el análisis de las respuestas obtenidas en los cuestionarios pasados en los congresos de los partidos permite apreciar, en determinados momentos, cómo son los militantes y, por lo tanto, qué semejanzas y qué diferencias hay con otros grupos de militantes en otros congresos. No obstante, el hecho que, referente a ERC, se disponga de una serie de datos de varios congresos, facilita la comparación, a la vez que proporciona algunas pistas referente a la dinámica sobre hacia dónde evoluciona y cómo lo hace la militancia de ERC. Los principales datos relativos a estas cuestiones se presentan en el apartado siguiente.

\section{Las características sociodemográficas de la militancia de ERC y sus opiniones políticas (1993-2004)}

El análisis comparativo-descriptivo de las principales características sociodemográficas de la militancia de ERC y de sus opiniones políticas para el periodo 1993-2004 se basa en los datos proporcionados por las respuestas obtenidas en cinco cuestionarios pasados en otros tantos congresos de ERC. Se trata de los congresos de 1993, 1996, 1998, 2001 y 2004. Mientras los cuatro primeros han sido ya analizados y los resultados obtenidos han sido publicados, los datos del 2004, el último congreso que ERC ha hecho, son inéditos. Además, la novedad reside en la presentación conjunta de las distintas variables para facilitar la comparación entre congresos.

En la tabla 5, se exponen los principales datos de estos congresos, entre los cuales se tiene que destacar no sólo el número de respuestas obtenidas en ellos ${ }^{8}$, sino también el porcentaje que significan sobre los asistentes y, asimismo, el porcentaje estimado sobre el conjunto de la militancia del partido.

En la presente nota de investigación, sólo se presentan, para la comparación en las sucesivas tablas, los datos de los congresos de 1993, 1998, 2001 y 2004. Se ha desechado la encuesta de 1996, porque se había utilizado un cuestionario tan diferente a los demás que dificultaba enormemente la comparación. De los cuatro cuestionarios finalmente utilizados, el primero y el último servirán para constatar los elementos de cambio y de continuidad, mientras que los otros dos apuntarán (o no) el proceso de cambio de un congreso al otro.

8. En todos los casos, el procedimiento para pasar el cuestionario fue el mismo. En la documentación que se entregaba al congresista, se incluía el cuestionario con preguntas cerradas (la mayoría). Desde la organización del congreso, se pedía que se respondiera a las preguntas y que se depositaran los cuestionarios rellenados en los lugares dispuestos a tal efecto. 
Tabla 5. Estudios sobre los asistentes a los congresos nacionales de ERC (1993-2004)

\begin{tabular}{lrrrrr}
\hline Año & $\mathbf{1 9 9 3}$ & $\mathbf{1 9 9 6}$ & $\mathbf{1 9 9 8}$ & $\mathbf{2 0 0 1}$ & $\mathbf{2 0 0 4}$ \\
Lugar & Barcelona & Vilafranca & Girona & Tarragona & Lleida \\
Núm. cuestionarios & $\mathbf{6 6 9}$ & $\mathbf{6 8 0}$ & $\mathbf{2 7 7}$ & $\mathbf{4 9 0}$ & $\mathbf{1 . 1 0 4}$ \\
\hline Votación más alta & 831 & 987 & 537 & 777 & 1.379 \\
\% respuestas & 80,51 & 68,90 & 51,58 & 63,06 & 80,05 \\
Núm. militantes ERC & c. 6.000 & c. 6.500 & 4.582 & 4.420 & 8.614 \\
\% cuestionarios/militantes & $11,2 \%$ & $10,5 \%$ & $6,0 \%$ & $11,1 \%$ & $12,8 \%$ \\
\% votación más alta/militantes & $13,9 \%$ & $15,2 \%$ & $11,7 \%$ & $17,6 \%$ & $16,0 \%$ \\
\hline
\end{tabular}

Fuentes:

Para 1993: Argelaguet, Maestro, Amorós (1998).

Para 1996: Argelaguet, Maestro (1999).

Para 1998 y 2001: Argelaguet (2004).

Para 2004: elaboración propia a partir de la explotación de las respuestas del cuestionario correspondiente.

La comparación entre 1993 y 2004 cobra más relevancia, no sólo por ser los dos casos extremos, sino por el hecho de tratarse de los congresos donde el número de respuestas es mayor y también es mayor el porcentaje de respuestas sobre la asistencia al congreso (superior al $80 \%$ en ambos casos) y sobre el conjunto de la militancia (más del $11 \%$ en ambos casos, también).

En las páginas siguientes, se presentarán varias tablas con la mayoría de los datos obtenidos en estos cuatro congresos, para que se puedan apreciar las similitudes y las diferencias entre ellos. Así mismo, cada tabla irá acompañada de un breve comentario de los elementos que se consideran dignos de ser subrayados.

En la tabla 6, se incluyen variables sociodemográficas: género; grupo de edad; lugar de nacimiento del encuestado, de su padre y de su madre, y origen familiar. En la tabla 7, se encuentran más variables que completan el perfil sociodemográfico: lugar de residencia, nivel de estudios, situación laboral y ocupación profesional. En la tabla 8, hay datos básicos sobre el uso lingüístico en la familia, con los amigos y en el lugar de trabajo. La tabla 9 está dedicada a la práctica religiosa, y la tabla 10 , al asociacionismo. Finalmente, la tabla 11 corresponde a los datos sobre la autoubicación del individuo y del partido en los ejes ideológicos y en la identidad nacional subjetiva, y las tablas 12 y 13 incluyen datos sobre la militancia política y actividades en el partido o en nombre del partido. En total, se trata de treinta variables distintas que permiten esbozar un retrato bastante completo (aunque sometido al sesgo del procedimiento de obtención de datos y a la veracidad de las respuestas proporcionadas) del núcleo militante más activo del partido decano de Cataluña entre 1993 y 2004.

La tabla 6, relativa a algunos datos sociodemográficos básicos de la militancia de ERC, muestra con claridad qué tipo de personas conforman el núcleo duro de los asistentes a los distintos congresos del partido. Se trata de una formación política donde los hombres son una clara mayoría, si bien la presen- 
cia de la mujer va penetrando lentamente. Aun así, en 2004, más de tres cuartas partes de las respuestas fueron de hombres.

La variable «grupos de edad» va acompañada del cálculo de la media de edad en los distintos congresos. Aquí se aprecia una clara evolución hacia un partido donde los que se sitúan alrededor de los cuarenta años son la mayoría. En efecto, el partido se ha ido «envejeciendo» relativamente: de 37,3 años en 1993 a 41,9 años en 2004. Otro cambio significativo ha sido la pérdida de peso de los grupos más jóvenes y, en correspondencia, el crecimiento de los grupos medios, que, eventualmente, indicaría la presencia de un cierto efecto de generación en la militancia de ERC.

Respecto al lugar de nacimiento del respondiente (o al de su padre y su madre), se tiene que hacer notar que, en ERC, es claramente mayoritario el grupo de los nacidos en Cataluña (o, por extensión, en el resto del dominio lingüístico del catalán): más del $96 \%$ en todos los años. Combinando estos datos con los del lugar de nacimiento de los progenitores, se ha calculado una nueva variable, el "origen familiar», con tres categorías: "autóctonos», «mixtos» e «inmigrantes». Dado que hay un $10-12 \%$ de personas cuyo padre o cuya madre (o ambos) han nacido fuera de los Países Catalanes, el porcentaje de «autóctonos» retrocede ligeramente hasta el $71,4 \%$ de 2004 , que contrasta con el $81 \%$ de 1993 . En cierto sentido, ERC, aunque continúa siendo un partido de «autóctonos», en los últimos años empieza a haber un cierto incremento de personas cuyos orígenes familiares se encuentran fuera de Cataluña.

En la tabla 7, hay una segunda batería de variables sociodemográficas respecto a las cuales se tiene que remarcar lo siguiente: ERC es un partido con una presencia en todos los tramos de tamaño de municipio y con una distribución que ha cambiado sólo en el sentido que ha crecido el porcentaje de militantes que viven en pequeños municipios. Cabe destacar que no llegan a la mitad los militantes que viven en poblaciones de más de 50.000 habitantes, lo que contrasta fuertemente con la distribución del conjunto de la población catalana.

En cuanto al nivel de estudios, la militancia de ERC manifiesta tenerlo considerablemente más alto que el conjunto de la población: los titulados universitarios (con grado medio o superior) superan siempre el $45 \%$ del total de los asistentes, llegando al 60\% en el último congreso. En cambio, el porcentaje de los que tienen estudios elementales o primarios es muy reducido desde 1993. Además, se ha ido disminuyendo desde entonces hasta el 3,9\% de la muestra de 2004.

Los datos sobre la situación laboral y la ocupación son bastante deficientes, por la razón de usar un cuestionario cerrado y sin posibilidad de ayudar al encuestado a responder adecuadamente. Sin embargo, cabe destacar el elevado porcentaje de empleados, tanto en el sector privado como en el público, y el bajo porcentaje de los que se definen como "trabajadores obreros». En cambio, el pequeño pero significativo porcentaje de los que se denominan "profesionales liberales» o "empresarios» completan el panorama que nos dibuja ERC como un partido claramente mesocrático, de clases medias ilustradas. 
Tabla 6. Variables sociodemográficas de la militancia de ERC (1)

$$
\begin{array}{ccccc}
\begin{array}{c}
\text { Barcelona } 1993 \\
(n=669)
\end{array} & (\mathrm{n}=680) & (\mathrm{n}=490) & (\mathrm{n}=1.104) & 2004-1993 \\
\hline
\end{array}
$$

\section{Género}

Hombre

82,1

17,9

75,8

Mujer

0,0

18,4

75,7
18,4
5,9

76,0

$-6,1$

$\mathrm{NC}$

5,8

5,9

23,6

$+5,7$

Grupo de edad

18-24

25-34

13,9

5,8

5,5

0,5

$+0,5$

35-50

\section{4,8}

33,9

33,3

44,4

51-64

8,5

10,8

65 y más

6,0

$\mathrm{NC}$

Media edad

3,4

3,2

1,8

37,26

38,85

Des. típica

13,59

11,35

5,5
31,4
45,3
9,4
5,3
3,1
39,85
12,13

$\begin{array}{cc}4,6 & -9,3 \\ 23,6 & -11,2 \\ 49,1 & +15,8 \\ 18,4 & +9,9 \\ 4,1 & -1,9 \\ 0,2 & -3,2 \\ 41,89 & +4,63 \\ 11,67 & -1,92\end{array}$

Lugar de nacimiento del encuestado

Cataluña

Resto PPCC ${ }^{1}$

94,6

2,4

96,0

2,2

Estado español

1,5

Extranjero

1,2

1,1

0,3

0,7

93,9

92,4

$-2,2$

4,3

4,1

$+1,7$

0,8

2,3

$+0,8$

NC

0,0

0,8

1,1

$-0,1$

0,2

0,1

$-0,2$

Lugar de nacimiento del padre

\begin{tabular}{lrrrrr} 
Cataluña & 86,2 & 84,8 & 81,2 & 81,9 & $-4,3$ \\
Resto PPCC & 4,2 & 4,7 & 6,1 & 5,3 & $+1,1$ \\
Estado español & 8,2 & 9,0 & 10,4 & 10,8 & $+2,6$ \\
Extranjero & 1,0 & 1,4 & 1,9 & 2,0 & $+1,0$ \\
NC & 0,3 & 0,0 & 0,4 & 1,0 & $+0,7$ \\
\hline Lugar de nacimiento de la madre & & & & \\
Cataluña & 82,7 & 85,9 & 83,3 & 80,1 & $-2,6$ \\
Resto PPCC & 3,7 & 4,0 & 5,7 & 5,0 & $+1,3$ \\
Estado español & 11,2 & 9,4 & 9,6 & 11,7 & $+0,5$ \\
Extranjero & 1,3 & 0,7 & 1,0 & 1,9 & $+0,6$ \\
NC & 1,0 & 0,0 & 0,4 & 0,4 & $-0,6$ \\
\hline Origen familiar & & & & & \\
Autóctono & 81,0 & 82,3 & 80,6 & 71,4 & $-9,6$ \\
Mixto & 13,8 & 14,8 & 15,1 & 16,8 & $+3,0$ \\
Inmigrante & 3,9 & 2,5 & 3,9 & 10,7 & $+6,8$ \\
NC & 1,3 & 0,4 & 0,4 & 1,2 & $-0,1$
\end{tabular}

1. «PPCC» equivale a «Países Catalanes», es decir, los nacidos en el País Valenciano, en las Islas Baleares y en la Cataluña Norte.

2. Remodificación a partir del lugar de nacimiento del encuestado, del de su padre y del de su madre. "Autóctono" son los nacidos en Cataluña (o en el resto de los Países Catalanes) con ambos progenitores también nacidos en Cataluña (o en el resto de los Países Catalanes). «Mixto» son los nacidos en Cataluña (o PPCC) y con un progenitor nacido en Cataluña. «Inmigrante» son las personas cuyos progenitores han nacido fuera de los PPCC. 
Tabla 7. Variables sociodemográficas de la militancia de ERC (2)

\section{Barcelona 1993 Girona 1998 Tarragona 2001 Lleida 2004 Dif.}

$(n=669) \quad(n=680) \quad(n=490) \quad(n=1.104) \quad 2004-1993$

Lugar de residencia

$<1.000$ habitantes

$1.001-5.000$

$<5.000$

- $\quad 6,5$

$5.001-20.000$

20.001-50.000

$\overline{14}, 6$

12,3

21,1

18,8

12,3

25,9

50.001-100.000

13,0

$>100.000$

13,4

Barcelona

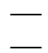

$>50.000$

12,6

15,9

NS/NC

41,9

51,4

0,4

3,9

15,3

19,2

6,5

21,6

12,9

14,7

13,7

16,7

45,1

0,6

1,2

Nivel de estudios

Elementales

y primarios
Bach. elem.,

EGB, FP1

8,8

4,7

5,7

3,9

$-4,9$

Formación

$$
\text { profesional }
$$

9,4

14,8

11,4

11,7

$+2,3$

8,8

Bach. sup., BUP, FP2 18,2

Técnico sin título $\quad 9,1$

Univ. grado medio $\quad 10,2$

Univ. grado superior $\quad 35,1$

NS/NC

0,3

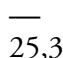

$\overline{24}, 3$

$-8,8$

25,3

24,2

-

31,0

18,2

39,8

21,5

$+6,9$

$20,1 \quad-1,0$

$12,9+0,6$

Situación laboral

Trabaja

Estudiante

74,9

0,0

0,6

$\begin{array}{rr}-\overline{23,1} & -8,8 \\ - & +4,9 \\ 18,4 & -9,1 \\ 42,8 & +8,2 \\ 0,1 & +7,7 \\ & -0,2\end{array}$

Parado/da

Jubilado/da

9,1

85,6

6,5

1,4

5,1

6,4

En casa

1,2

Otra

1,5

$\mathrm{NS} / \mathrm{NC}$

1,2

2,9

1,8

0,0

1,8

88,4

5,1

1,4

2,9

1,0

0,0

Ocupación

Profesión liberal

Empresario

11,2

5,6

10,8

3,2

10,2

4,5

10,9

$-0,3$

Empleado sector privado

$\overline{15,8}$

28,5

30,8

33,9

Administrativo/va

12,4

Profesor/a-maestro/a

Vendedor/

representante

Ingeniero/a

Empleado sector público

Autónomo

Campesino

Trabajador, obrero

Comerciante

Otra

6,6
3,4

10,8

27,4

1,4

15,0

3,6

13,5

8,7

27,6

5,7

3,5

0,7

4,9

0,4

0,4

(n)

2,5

0,8

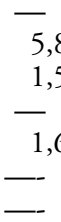

5,8

$-3,3$

$1,5 \quad-3,6$

$1,6 \quad+0,4$

1,2

$-0,2$

(237)

31,1

$+20,3$

5,9

$1,4 \quad 0,0$

$2,6 \quad-12,4$

6,0

$-7,5$

(433) 
Tabla 8. Usos lingüísticos de la militancia de ERC

\section{Barcelona 1993 Girona 1998 Tarragona 2001 Lleida 2004 Dif.}

$\begin{array}{llll}(n=669) & (n=680) & (n=490) & (n=1.104)\end{array} 2004-1993$

\section{Lengua en casa}

Catalán

Español

95,8

2,1

Catalán y español

1,0

94,6

93,1

$94,5 \quad-1,3$

Aranés

Otra lengua

0,1

0,3

1,4

2,2

2,9

$2,0 \quad-0,1$

Catalán y

otras lenguas

$\mathrm{NS} / \mathrm{NC}$

$-0,6 \quad 0,0$

0,0
0,4

0,6

1,0

$2,7+1,7$

$\begin{array}{llll}0,0 & - & - & -0,1 \\ 0,2 & 1,2 & +0,9\end{array}$

Lengua en el trabajo

Catalán

Español

85,8

1,9

Catalán y español

Aranés

Otra lengua

Catalán y

otras lenguas

$\mathrm{NS} / \mathrm{NC}$
4,8

0,1

1,3
6,0
76,2

1,4

18,4

- 0,0

0,0

4,0
74,9

1,0

16,3

$-0,6$

$\overline{0}, 4 \quad \overline{-0}, 2$

Lengua con amistades

Catalán

Español

91,5

0,7

90,6

0,0

2,9

$\overline{8,3}^{0,0}$

4,2

$\begin{array}{rr}84,1 & -1,7 \\ 4,2 & +2,3 \\ 11,2 & +6,4 \\ - & -0,1 \\ 1,1 & -0,2\end{array}$

Catalán y español

Aranés

3,7

0,1

Otra lengua

1,5

88,4

0,0

$2,5-3,5$

Catalán y

otras lenguas

NS/NC

$-\quad 0,0$

8,4

-

0,6

2,7
$-1,0$

$\begin{array}{rr}90,5 & -1,0 \\ 1,9 & +1,2 \\ 6,9 & +3,2 \\ - & -0,1 \\ 0,5 & -1,0\end{array}$

$1,6 \quad-0,8$

En la tabla 8, se presenta una pequeña incursión hacia los usos lingüísticos de la militancia en tres ámbitos diferentes: en la familia, con los amigos y en el trabajo. La presencia del uso del catalán es clarísimamente dominante en los tres ámbitos, siendo mayor en la familia, con más del $93 \%$ en todos los años. Por el contrario, en la familia, la presencia del español es minoritaria, y de manera constante. El uso del catalán en el trabajo - sobre todo- y con los amigos baja relativamente, aunque continúa siendo la lengua predominante. El leve retroceso del uso del catalán en estos ámbitos se relaciona con el aumento de los que optan por la respuesta «bilingüe». Finalmente, se tiene que destacar que no hay cambios significativos entre los dos años extremos, excepto una mayor presencia del español en el ámbito del trabajo.

La práctica religiosa está referenciada en la tabla 9. Desde 1993, el grupo mayoritario es el de "no creyentes» (siempre por encima del 40\%). Los que se califican de «ateos» superan siempre el $18 \%$ del total y están casi empatados 
Tabla 9. Práctica religiosa de la militancia de ERC

\begin{tabular}{|c|c|c|c|c|c|}
\hline & $\begin{array}{c}\text { Barcelona } 199 \\
\quad(n=669)\end{array}$ & $\begin{array}{c}3 \text { Girona } 1998 \\
(\mathrm{n}=680)\end{array}$ & $\begin{array}{l}8 \text { Tarragona } 2001 \\
(\mathrm{n}=490)\end{array}$ & $\begin{array}{l}\text { Lleida } 2004 \\
(\mathrm{n}=1.104)\end{array}$ & $\begin{array}{c}\text { Dif. } \\
2004-1993\end{array}$ \\
\hline $\begin{array}{l}\text { Católico } \\
\text { practicante }\end{array}$ & 8,5 & 6,1 & 5,3 & 5,8 & $-2,7$ \\
\hline Católico & 326 & 274 & 255 & 237 & -89 \\
\hline Otras religiones & 2,4 & 2,5 & 2,2 & 2,1 & $-0,3$ \\
\hline No creyente & 49,0 & 43,3 & 40,8 & 42,2 & $-6,8$ \\
\hline Ateo & - & 18,4 & 22,9 & 22,9 & $+22,9$ \\
\hline Otras respuestas & 2,3 & - & - & - & - \\
\hline $\mathrm{NS} / \mathrm{NC}$ & 5,1 & 2,2 & 3,3 & 3,3 & $-1,8$ \\
\hline
\end{tabular}

con los «católicos no practicantes». La militancia de ERC registra una clara evolución hacia posiciones alejadas de la Iglesia católica. Mientras en 1993, el $49 \%$ se decía «no creyente» (y no había la categoría de «ateo»), en 2004, la suma de «no creyentes» $(42,2 \%)$ y de «ateos» $(22,9 \%)$ llegaba al $65,1 \%$ del total de respuestas.

En la tabla 10, se incluyen datos sobre el asociacionismo de la militancia de ERC. La distribución entre varios tipos de asociación es bastante parecida a lo largo de los años. Se tiene que destacar el elevado asociacionismo en el ámbito cultural: en 2004, el 50\% dice ser miembro de una asociación cultural. En contraste, sólo un 20-25\% de los congresistas — según los años- está afiliado a un sindicato. Los sindicatos con más apoyo son, significativamente, los dos de ámbito español: UGT y CCOO.

En la tabla 11, se reflejan los datos sobre la autoubicación del encuestado en el eje ideológico y en el eje relativo a la identidad nacional subjetiva. En el primero, con siete posiciones, se ubica el individuo en la escala derecha e izquierda. En el segundo, con cinco posiciones, se ubica el individuo según su respuesta a la contraposición entre «español» y «catalán».

Las respuestas obtenidas en ambas escalas no son contraintuitivas en un partido independentista catalán que se define de izquierdas. En efecto, la inmensa mayoría de los militantes se autoubican en posiciones de izquierda y casi todos ellos en posiciones de identidad «sólo catalana».

Más interesante es la ubicación que hacen los encuestados del partido en estos dos mismos ejes. En términos generales, y lógicamente, ubican también el partido en posiciones izquierdistas y en posiciones de identidad «sólo catalana». No obstante, crece un poco el porcentaje de los que no saben o no quieren ubicar el partido en estos dos ejes (en especial, en el eje izquierda-derecha). Se puede destacar que, entre 1993 y 2004, la ubicación media en el eje ideológico se ha desplazado un poco hacia posiciones menos izquierdistas, lo cual contrasta con la apuesta política de impulsar un gobierno de izquierdas en Cataluña.

En la tabla 12 y 13, se incluyen los datos sobre la vinculación de la militancia con el partido o su actividad dentro del mismo. Así, ERC es un partido 
Tabla 10. Asociacionismo de la militancia de ERC

\begin{tabular}{|c|c|c|c|c|c|}
\hline $\begin{array}{r}\text { Barc } \\
(n\end{array}$ & \multicolumn{4}{|c|}{ Barcelona 1993 Girona 1998 Tarragona 2001 Lleida 2004} & $\begin{array}{c}\text { Dif. } \\
\text { 2004-1993 }\end{array}$ \\
\hline \multicolumn{6}{|l|}{ Tipo de asociación } \\
\hline Empresarial & 4,0 & 3,6 & 2,9 & - & 一 \\
\hline Sindical & 21,1 & 23,8 & 25,1 & 23,7 & $+2,6$ \\
\hline Vecinal & 15,2 & 17,7 & 17,6 & 14,2 & $-1,0$ \\
\hline Religiosa & 2,4 & 1,4 & 2,0 & 2,4 & 0,0 \\
\hline Cultural & 43,3 & 51,6 & 52,0 & 50,0 & $+6,7$ \\
\hline Ecologista & 12,4 & 16,2 & 15,5 & 12,7 & $+0,3$ \\
\hline Feminista & 1,6 & 1,8 & 1,0 & 2,4 & $+0,8$ \\
\hline Solidaria & 一 & 20,6 & 20,4 & 20,6 & - \\
\hline Deportiva & 30,9 & 34,7 & 27,3 & 33,1 & $+2,2$ \\
\hline Colegio profesional & 16,6 & 19,5 & 23,7 & 21,7 & $+5,1$ \\
\hline Cooperativa & 4,9 & 8,3 & 10,0 & 7,5 & $+2,6$ \\
\hline Otras & 13,5 & 15,9 & 12,2 & 7,4 & $-6,1$ \\
\hline \multicolumn{6}{|l|}{ Sindicato } \\
\hline UGT & - & 17,9 & 26,0 & 22,5 & - \\
\hline $\mathrm{CCOO}$ & - & 25,4 & 24,4 & 31,7 & - \\
\hline CGT & - & 4,5 & 4,1 & 0,0 & - \\
\hline USTEC & - & 17,9 & 9,8 & 9,9 & - \\
\hline BEI & - & 6,0 & 1,6 & 0,0 & - \\
\hline CSC & - & 4,5 & 5,7 & 8,4 & - \\
\hline Unió de Pagesos & - & 4,5 & 10,6 & 3,8 & - \\
\hline Otros & - & 19,4 & 15,4 & 0,0 & - \\
\hline NC & - & - & 2,4 & - & - \\
\hline$(n)$ & - & $(67)$ & $(123)$ & $(262)$ & - \\
\hline
\end{tabular}

cuya gran mayoría de miembros no ha militado anteriormente en otros partidos. No obstante, en 1993, cerca de un tercio sí lo había hecho. Este dado concuerda, sin problemas, con la historia reciente de ERC como un partido donde va a parar gente que había militado en otras formaciones de este mismo espacio político y al que acuden cuando este partido se «refunda» en 1989.

La percepción que ERC es un partido en constante renovación se refuerza con los datos sobre los años en que uno se afilió o, alternativamente, los años de militancia de cada uno. En efecto, en todos los congresos, el grupo más numeroso es aquél que dice tener una afiliación reciente o muy reciente. Así, el dato más espectacular se da en 2004, donde el 31,9\% dice tener menos de un año de militancia. Si en este congreso se debatió la pertinencia del pacto firmado en 2003, es de esperar que, de entrada, un tercio de los asistentes le diera apoyo, ya que se inscribió en el partido después de realizarse aquél. Si a este porcentaje se añade que el segundo grupo más numeroso, con el $27,8 \%$, es el que lleva de dos a cinco años militando, se puede decir que la dirección política de 2004 puede contar con un apoyo más que sólido: más del 60\% se ha hecho militante durante su mandato. 
Tabla 11. Ubicación de la militancia de ERC y del partido en los ejes ideológico y nacional

\section{Barcelona 1993 Girona 1998 Tarragona 2001 Lleida 2004 Dif.}

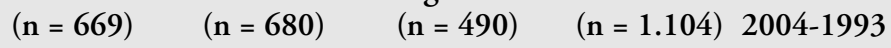

Autoubicación en el eje izquierda-derecha ${ }^{1}$

$\begin{array}{lrrrrr}\text { Ext. izquierda (1) } & 28,3 & 6,1 & 8,6 & 7,9 & -20,4 \\ \text { Izquierda (2) } & 49,8 & 67,1 & 68,6 & 69,1 & +19,3 \\ \text { Centro izq. (3) } & 10,6 & 24,5 & 19,8 & 19,5 & +8,9 \\ \text { Centro (4) } & 4,3 & 1,8 & 1,2 & 1,4 & -2,9 \\ \text { Centro derecha (5) } & 0,3 & 0,0 & 0,0 & 0,1 & -0,2 \\ \text { Derecha (6) } & 1,3 & 0,0 & 0,0 & 0,1 & -1,2 \\ \text { Extrema derecha (7) } & 0,1 & 0,0 & 0,0 & 0,0 & -0,1 \\ \text { NS/NC } & 5,2 & 0,4 & 1,8 & 1,9 & -3,3 \\ \text { Media } & 1,98 & 2,22 & 2,14 & 2,27 & +0,29 \\ \text { Desviación típica } & 0,94 & 0,58 & 0,57 & 0,99 & +0,05\end{array}$

Autoubicación en el eje nacional

\begin{tabular}{llccc}
\hline Sólo español (1) & - & 0,0 & 0,0 & 0,5 \\
Esp. > cat. (2) & - & 0,0 & 0,0 & 0,4 \\
Esp. = cat. (3) & - & 0,0 & 0,0 & 0,4 \\
Catalán > esp. (4) & - & 3,2 & 4,7 & 5,5 \\
Sólo catalán (5) & - & 96,4 & 94,7 & 91,7 \\
NS/NC & - & 0,4 & 0,6 & 1,5 \\
Media & - & 4,97 & 4,95 & 4,92 \\
Desviación típica & - & 0,18 & 0,21 & 0,45
\end{tabular}

Ubicación de ERC en el eje izquierda-derecha ${ }^{1}$

$\begin{array}{lrrrrr}\text { Ext. izquierda (1) } & 17,6 & 0,4 & 0,6 & 1,0 & -16,6 \\ \text { Izquierda (2) } & 51,0 & 58,8 & 50,4 & 57,2 & +6,2 \\ \text { Centro izq. (3) } & 16,7 & 31,0 & 35,1 & 32,9 & +16,2 \\ \text { Centro (4) } & 8,8 & 1,4 & 1,8 & 1,0 & -7,8 \\ \text { Centro derecha (5) } & 1,3 & 0,0 & 0,2 & 0,1 & -1,2 \\ \text { Derecha (6) } & 0,7 & 0,0 & 0,0 & 0,2 & -0,5 \\ \text { Extrema derecha (7) } & 0,1 & 0,0 & 0,0 & 0,0 & -0,1 \\ \text { NS/NC } & 3,6 & 8,3 & 11,8 & 7,7 & +4,1 \\ \text { Media } & 2,26 & 2,37 & 2,44 & 2,81 & +0,55 \\ \text { Desviación típica } & 0,86 & 0,52 & 0,56 & 1,59 & +0,73\end{array}$

Ubicación de ERC en el eje nacional

$\begin{array}{llccc}\text { Sólo español (1) } & - & 0,0 & 0,0 & 0,3 \\ \text { Esp. > cat. (2) } & - & 0,0 & 0,0 & 0,3 \\ \text { Esp. = cat. (3) } & - & 0,0 & 0,2 & 0,0 \\ \text { Catalán > esp. (4) } & - & 3,6 & 6,1 & 8,9 \\ \text { Sólo catalán (5) } & - & 92,4 & 84,5 & 83,0 \\ \text { NS/NC } & - & 4,0 & 9,2 & 7,6 \\ \text { Media } & - & 4,96 & 4,93 & 4,97 \\ \text { Desviación típica } & - & 0,19 & 0,27 & 0,47\end{array}$

1. Para el año 1993, la escala era de diez posiciones. Se ha recodificado en siete posiciones para posibilitar la comparación con otros años: 1,$1 ; 2-3,2 ; 4,3 ; 5-6,4 ; 7,5 ; 8-9,6 ; 10,7$. 
Tabla 12. Vinculación de la militancia de ERC con el partido (1)

\begin{tabular}{|c|c|c|c|c|c|}
\hline Barc & $\begin{array}{l}\text { rcelona } 199 \\
(\mathrm{n}=669)\end{array}$ & $\begin{array}{l}\text { Girona } 1998 \\
(\mathrm{n}=680)\end{array}$ & $\begin{array}{l}\text { Tarragona } 2001 \\
\quad(\mathrm{n}=490)\end{array}$ & $\begin{array}{l}\text { Lleida } 2004 \\
(\mathrm{n}=1.104)\end{array}$ & $\begin{array}{c}\text { Dif. } \\
2004-1993\end{array}$ \\
\hline \multicolumn{6}{|l|}{ Militancia anterior } \\
\hline Sí & 32,5 & 23,1 & 24,1 & 20,7 & $-11,8$ \\
\hline No & 67,5 & 76,9 & 74,9 & 79,3 & $+11,8$ \\
\hline $\mathrm{NS} / \mathrm{NC}$ & 0,0 & 0,0 & 1,0 & 0,0 & 0,0 \\
\hline \multicolumn{6}{|c|}{ Organización anterior } \\
\hline Independ. varios & 42,9 & 44,6 & 35,3 & 15,7 & \\
\hline Crida Solidaritat & 9,2 & & & & \\
\hline MDT & 16,6 & 7,7 & 10,9 & 8,7 & \\
\hline PSAN & 0,0 & 7,7 & 5,0 & 5,7 & \\
\hline Catalunya Lliure & 12,9 & 6,2 & 7,6 & 6,6 & \\
\hline CDC-CiU & 7,8 & 0,0 & 4,2 & 9,6 & \\
\hline PSC & 10,1 & 4,6 & 9,2 & 0,0 & \\
\hline PSUC/ICV & 6,5 & 9,2 & 12,6 & 10,0 & \\
\hline Otros izquierda & 8,8 & 15,4 & 7,6 & 1,3 & \\
\hline UPV & 1,4 & 1,5 & 1,7 & 0,0 & \\
\hline Verds & 0,0 & 0,0 & 0,8 & 0,0 & \\
\hline Otros & 7,8 & 0,0 & 1,7 & 28,8 & \\
\hline ERC & 0,0 & 0,0 & 0,8 & 0,0 & \\
\hline Acción Catalana & 0,0 & 0,0 & 0,0 & 0,9 & \\
\hline Assemblea Cat. & 0,0 & 0,0 & 0,0 & 0,4 & \\
\hline NS/NC & 0,0 & 3,1 & 2,5 & 0,0 & \\
\hline$(n)$ & $(317)$ & $(65)$ & $(119)$ & $(229)$ & \\
\hline \multicolumn{6}{|c|}{ Grupo año de afiliación } \\
\hline Antes de 1976 & 4,2 & 5,4 & 0,4 & 2,5 & \\
\hline 1977-1986 & 11,4 & 6,5 & 3,3 & 4,7 & \\
\hline 1987-1989 & 23,0 & 11,9 & 7,3 & 5,7 & \\
\hline $1990-1995$ & 58,0 & 57,8 & 38,8 & 18,8 & \\
\hline $1996-2003$ & - & 18,4 & 43,5 & 55,7 & \\
\hline De 2004 en adelante & e - & - & - & 12,6 & \\
\hline NS/NC & 3,4 & - & 6,7 & 0,0 & \\
\hline \multicolumn{6}{|l|}{ Años de militancia } \\
\hline 0 a 1 años & 27,4 & 6,5 & 17,8 & 31,9 & $+4,5$ \\
\hline 2 a 5 años & 44,8 & 35,0 & 25,7 & 27,8 & $-17,0$ \\
\hline 6 a 10 años & 13,2 & 43,7 & 33,3 & 13,0 & $-0,2$ \\
\hline 11 a 15 años & 6,0 & 4,7 & 13,5 & 16,4 & $+10,4$ \\
\hline 16 a 20 años & 3,9 & 4,0 & 0,8 & 5,3 & $+1,4$ \\
\hline 21 a 25 años & 1,6 & 2,5 & 2,2 & 3,8 & $+2,2$ \\
\hline NS/NC & 3,1 & 3,6 & 6,7 & 1,9 & $-1,2$ \\
\hline Media & 5,00 & 6,48 & 6,39 & 6,24 & $+1,24$ \\
\hline Desviación típica & 8,29 & 4,91 & 4,89 & 6,65 & $-1,64$ \\
\hline
\end{tabular}


Tabla 13. Vinculación de la militancia de ERC con el partido (2)

\begin{tabular}{|c|c|c|c|c|c|}
\hline & $\begin{array}{c}\text { Barcelona 19) } \\
\quad(n=669)\end{array}$ & $\begin{array}{l}\text { Girona } 1998 \\
(n=680)\end{array}$ & $\begin{array}{l}\text { Tarragona } 2001 \\
(\mathrm{n}=490)\end{array}$ & $\begin{array}{l}\text { Lleida } 2004 \\
(\mathrm{n}=1.104)\end{array}$ & $\begin{array}{c}\text { Dif. } \\
\text { 2004-1993 }\end{array}$ \\
\hline \multicolumn{6}{|c|}{ Asistencia a congresos ERC } \\
\hline Sí & 59,0 & 80,1 & 54,9 & 43,4 & $-15,6$ \\
\hline & 41,0 & 19,9 & 44,5 & 55,8 & 14,8 \\
\hline NS/NC & - & - & 0,6 & 0,8 & $+0,8$ \\
\hline \multicolumn{6}{|c|}{ Congresos nacionales anteriores } \\
\hline Lleida 1989 & $\begin{array}{c}37,0 \\
(146)\end{array}$ & $\begin{array}{l}52,5 \\
(61)\end{array}$ & $\begin{array}{l}47,6 \\
(63)\end{array}$ & $\begin{array}{c}7,0 \\
(77)\end{array}$ & \\
\hline \multirow[t]{2}{*}{ Barcelona 1991} & 65,3 & 69,8 & 58,2 & 9,0 & \\
\hline & $(258)$ & $(96)$ & $(110)$ & (99) & \\
\hline Vic 1992 & $\begin{array}{c}60,5 \\
(239)\end{array}$ & $\begin{array}{c}39,5 \\
(152)\end{array}$ & $\begin{array}{c}52,8 \\
(142)\end{array}$ & $\begin{array}{c}8,9 \\
(98)\end{array}$ & \\
\hline \multirow[t]{2}{*}{ Barcelona 1993} & - & 67,3 & 63,1 & 13,6 & \\
\hline & - & $(168)$ & (179) & $(150)$ & \\
\hline \multirow[t]{2}{*}{ Manlleu 1995} & - & 64,7 & 63,4 & 16,4 & \\
\hline & - & $(207)$ & $(235)$ & $(181)$ & \\
\hline \multirow[t]{2}{*}{ Vilafranca 1996} & - & 83,1 & 73,0 & 23,1 & \\
\hline & - & (236) & $(282)$ & $(255)$ & \\
\hline \multirow[t]{2}{*}{ Girona 1998} & - & - & 64,3 & 16,8 & \\
\hline & - & - & (308) & $(185)$ & \\
\hline \multirow[t]{2}{*}{ Tarragona 2001} & - & - & - & 33,6 & \\
\hline & - & - & - & $(371)$ & \\
\hline \multicolumn{6}{|c|}{ Responsabilidades dentro de ERC } \\
\hline Sí & 51,3 & 63,2 & 52,2 & 45,2 & $-6,1$ \\
\hline No & 48,7 & 36,8 & 47,1 & 52,1 & $+3,4$ \\
\hline NS/NC & - & - & 0,6 & 2,7 & $+2,7$ \\
\hline \multicolumn{6}{|c|}{ Nivel de responsabilidad interna } \\
\hline Ejec. nacional & 23,0 & 5,7 & 5,5 & 2,8 & $-10,6$ \\
\hline Consell Nacional & 28,0 & 24,2 & 9,6 & & \\
\hline Ejec. regional & 11,4 & 9,7 & 15,2 & 9,4 & $-2,0$ \\
\hline Ejec. comarcal & 32,1 & 27,4 & 24,2 & 28,1 & $-4,0$ \\
\hline Ejec. local & 73,8 & 73,1 & 70,7 & 76,8 & $+3,0$ \\
\hline \multirow{2}{*}{$\begin{array}{l}\text { Otra } \\
(n)\end{array}$} & - & 2,9 & 2,0 & 1,4 & $+1,4$ \\
\hline & (343) & $(175)$ & $(256)$ & (499) & \\
\hline
\end{tabular}

Cargo en representación de ERC

Sí

No

$\mathrm{NS} / \mathrm{NC}$

Tipos de cargo

Concejal

Alcalde

Consejero comarcal -

Diputado

Consejo de admón.

Otro cargo

$(n)$

$\begin{array}{lr}- & 15,2 \\ - & 84,1 \\ - & 0,7\end{array}$

0,7

73,8

7,1

19,0

2,4

4,8

4,8

(42)
19,0

25,7

81,0

72,0

2,3 
La tabla 13 recoge más datos sobre la vinculación de la militancia de ERC con el partido: asistencia a congresos anteriores de ERC, a qué congresos, si se tienen o no responsabilidades dentro del partido y a qué nivel, si se tiene un cargo político en representación de ERC y, finalmente, cuál es este cargo.

En cuanto a la asistencia a congresos anteriores, el dato más significativo es que en todos los congresos, excepto el último, son mayoría los que ya han asistido a otros celebrados antes. La novedad del 2004 se inscribe en el proceso de incorporación de nueva militancia a raíz de los buenos resultados del 2003 y del 2004.

En los congresos de 1993, 1998 y 2001, hay una mayoría de asistentes que dice tener responsabilidades orgánicas en ERC. En 2004, en cambio, ya son una minoría, pero sin duda aún importante. El nivel de responsabilidad es en el ámbito local: siempre supera el $70 \%$.

Se puede destacar que el porcentaje de los que tienen cargos de representación en ERC va aumentando a lo largo de los congresos, en consonancia con la mejora de los resultados electorales en las distintas convocatorias. Si en 1998 era el 15,2\% de los congresistas, en 2004 se alcanzaba la cifra del 25,7\%. En todos los años, los cargos de representación más presentes era en el ámbito local: concejal o alcalde.

\section{Conclusiones generales}

De los datos anteriormente expuestos, se pueden extraer las siguientes conclusiones sobre el perfil de la militancia de ERC y de sus elementos de cambio y de continuidad. Se trata de un partido pequeño, con unos pocos miles de afiliados. La evolución del número de miembros está en clara correspondencia con la evolución electoral del mismo y, en términos más generales, con los avatares por los que pasa el partido.

El perfil medio del militante de ERC, según las respuestas obtenidas entre 1993 y 2004, no ha cambiado excesivamente. ERC es un partido donde predomina el elemento masculino, de mediana edad, de personas nacidas en Cataluña y con ambos progenitores también nacidos en Cataluña, mayoritariamente catalanohablantes, desvinculados de la religión católica en un porcentaje elevado, con un nivel de instrucción alto (educación universitaria), que trabaja de asalariado tanto en el sector privado como en el público y que se considera de izquierdas con una identidad exclusiva catalana.

La militancia en el partido es reciente, no acostumbra a tener una previa y, si la tiene, procede de organizaciones de carácter independentista diverso. Los asistentes al congreso son, en su mayoría, militantes de base, pero hay un núcleo importante (y creciente a medida que el partido va consiguiendo mejores resultados electorales) que tiene cargos de representación, sobre todo en el ámbito local.

Finalmente, los datos confirman que ERC es un partido que va cambiando su base humana a lo largo de los años, si bien lo hace dentro de un mismo perfil sociodemográfico e ideológico. 


\section{Bibliografía}

AlqUÉZAR, Ramon y otros (2001). Esquerra Republicana de Catalunya. 70 anys d'història. Barcelona: Columna.

ARgelaguet, Jordi (2004). «L'Esquerra Republicana de Catalunya: la troisième force de l'espace politique catalan». Pôle Sud, n. ${ }^{\circ} 20$, p. 9-24.

- (2004). «Els congressos nacionals d'Esquerra Republicana de Catalunya (Girona 1998 i Tarragona 2001)». En: Montserrat BARAS (ed.) (2004). Els militants dels partits polítics a Catalunya. Perfils socials i percepcions polítiques. Barcelona: Institut de Ciències Polítiques i Socials. Col-lecció Barcelona, n. ${ }^{\circ} 18$.

Argelaguet, Jordi; Maestro, Jesús; Amorós, Moisés (1998). «Anàlisi sociològica dels assistents al XIX Congrés Nacional d'ERC (Barcelona, 1993)». Papers, n. ${ }^{\circ} 54$, p. 187-200.

ARgelaguet, Jordi; MAeSTRO, Jesús (1999). «XXI Congrés d'Esquerra Republicana de Catalunya». En: Jordi SÀnCHEZ (ed.) (1999). Estudis de les elits dels partits polítics de Catalunya. Barcelona: Institut de Ciències Polítiques i Socials.

IVern, Maria Dolors (1988). Esquerra Republicana de Catalunya (1931-1936). Montserrat: Abadia de Montserrat.

LUCAS, Manel (2004). ERC. La llarga marxa: 1977-2004. De la il.legalitat al govern. Barcelona: Columna.

Molas, Isidre (ed.) (2000). Diccionari dels partits politics de Catalunya. Segle XX. Barcelona: Enciclopedia Catalana.

Montero, José Ramón; GunTHer, Richard; Linz, Juan J. (eds.) (2007). Partidos politicos. Viejos conceptos y nuevos retos. Madrid: Fundación Alfonso Martín Escudero y Editorial Trotta.

SAlLÉs, Anna (1986). Quan Catalunya era d'esquerra. Barcelona: Edicions 62.

VERGE, Tània (2007). «Representación política y modelos de partidos en España: los casos de IU, PSOE y PP». En: José Ramón MONTERO, Richard GunTHER y Juan J. LINZ. Partidos políticos. Viejos conceptos y nuevos retos. Madrid: Fundación Alfonso Martín Escudero y Editorial Trotta. 